\title{
Optimal Integration of the Renewable Energy to the Grid by Considering Small Signal Stability Constraint
}

\author{
I Made Wartana ${ }^{1}$, Ni Putu Agustini' ${ }^{2}$ Jai Govind Singh $^{3}$ \\ ${ }^{1,2}$ Department of Electrical Engineering, National Institute of Technology (ITN) Malang \\ ${ }^{3}$ Department of Energy, Environment and Climate Change, SERD, Asian Institute of Technology (AIT) Thailand
}

\begin{abstract}
Article Info
\section{Article history:}

Received Jan 24, 2017

Revised May 4, 2017

Accepted Jun 10, 2017

\section{Keywords:}

Eigenvalue

Optimal integration

Renewable energy

Security

Stability

ABSTRACT

In recent decades, one of the main management's concerns of professional engineers is the optimal integration of various types of renewable energy to the grid. This paper discusses the optimal allocation of one type of renewable energy i.e. wind turbine to the grid for enhancing network's performance. A multi-objective function is used as indexes of the system's performance, such as increasing system loadability and minimizing the loss of real power transmission line by considering security and stability of systems' constraints viz.: voltage and line margins, and eigenvalues as well which is representing as small signal stability. To solve the optimization problems, a new method has been developed using a novel variant of the Genetic Algorithm (GA), specifically known as Non-dominated Sorting Genetic Algorithm II (NSGAII). Whereas the Fuzzy-based mechanism is used to support the decision makers prefer the best compromise solution from the Pareto front. The effectiveness of the developed method has been established on a modified IEEE 14-bus system with wind turbine system, and their simulation results showed that the dynamic performance of the power system can be effectively improved by considering the stability and security of the system.
\end{abstract}

Copyright $\odot 2017$ Institute of Advanced Engineering and Science. All rights reserved.

\section{Corresponding Author:}

I Made Wartana,

Department of Electrical Engineering,

National Institute of Technology (ITN) Malang,

Jl. Bend. Sigura-gura No. 2 Malang, East-Java, Indonesia.

Email: m.wartana@lecturer.itn.ac.id

\section{INTRODUCTION}

The growing awareness of environmental issues and the efforts to reduce dependency on fossil fuel resources are bringing renewable energy resources to the mainstream power sector. Among the various renewable resources, wind power is assumed to have the most commercial technics and economic prospects. The integration of renewable energy which is represented as a Distributed Generation (DG) to the grid has received wide attention and scope in power system for several reasons [1]. First, DG helps to utilize the distributed but with small energy resources. Second, reducing the use of transmission capacity as most DG is located near the center of the load along with several types of DG also provide reactive power to support the power system. Third, as DG is located close to the load, thus it reduces transmission loss and at the same time improving system performance. The fourth advantage is to delay the investment in transmission lines and construction of large power plants. Above all, second option/advantage has been utilized in this chapter to enhance the system loadability by optimal placement of DGs in the network. However, some DGs, particularly wind generation system do not produce or almost negligible reactive power generate. Therefore, several other mechanisms have been used to compensate the reactive power requirement when DG did not generate/support. 
Conversely, in recent years, integration of a wide variety of Distributed Generation (DG) technology in distribution networks has become one of the major management concerns for professional engineers. Some of the major technical benefits are improving voltage profile by reducing active power losses, enhancing system security and reliability for power quality improvement, increasing overall energy efficiency [2], relieving transmission, and distribution congestion. In addition, DGs are used near load center and so, it utilizes the available small energy resources very efficiently and effectively and, hence, improves the energy access as well [3]. Moreover, it also helps to secure and restore the network operation during emergency and/or after blackout. However, integration of a wide variety of DG technology in distribution networks has become one of the major management concerns for operational people.

Numerous works were made on the optimal allocation of DG for some different purposes. Different approach techniques have been suggested i.e., Genetic Algorithm (GA) [2], Quantum GA [4], a simple conventional iterative search technique [5] for optimal location and settings of multi-types of DG. [6] The optimal DG placement has been compared using CSA, GSA, PSO and GA for minimum real power loss in radial distribution system. However, the system stability and security constraints have not exclusively considered yet for maximizing the system loadability within any condition of the grid and their impact on the transmission loss with the DG placement.

From these literature works, it can be observed that most of the problems in optimal DG locations are often disclosed separately as a matter of mono-objective optimization [2],[4],[5], [6]. Awkwardly, the formulation of the problems as a mono-objective optimization is not quite practical. However, it is always good to utilize optimal DG placement with two conflicting objectives taking into account the security and stability of the system formulated as a multi-objective problem and solved simultaneously.

In this work, a multi-objective problem has been formulated for maximizing the system loadability by optimal location and settings of a DG, viz. wind generation system or farm while maintaining the system security and stability margin within acceptable range. By means of DG optimal placement, the system loadability has been maximized whereas the active power loss of the transmission line was minimized. The multi-objective problems have been solved simultaneously using the novel variant of GA specialized in multi-objective optimizations problem, namely the NSGA-II by optimal location and sizing of the DG. The suggested methodology has been effectively investigated on IEEE 14-bus systems and the results have been compared with literature work [4], [5], and [7].

\section{RESEARCH METHOD}

\subsection{Modelling of $D G$}

In this paper, one type of the wind turbines namely DFIG has been engaged which is applied for the energy conversion, called as variable speed systems, the power electronic interface which is used to connect the DG with utility, also provides some reactive power support [8]. The DFIG is a wound rotor induction generator with a voltage source converter connected to the slip-rings of the rotor. The stator winding is coupled directly to the grid and the rotor winding is connected to the grid via a power electronic converter. References [9] provide a detailed description of the operation of a DFIG which is incoporeted in Power System Analysis Toolbox (PSAT).

\subsection{Problem Formulation}

As indicated, the goal of the stated optimization problem is the optimal placements of DG into power network in order to maximize the loadability and security margin, and to minimize the real power loss in transmission lines. The optimal location and sizing of DG is formulated as a real constrained mixed discrete continuous multi-objective optimization problem.

Therefore, the presented problem becomes a multi-objective optimization problem that has two objective functions to optimize simultaneously, which can be denoted as:

$$
\begin{aligned}
& \text { Minimize } F(\mathbf{x}, \mathbf{u})=\left[F_{1}(\mathbf{x}, \mathbf{u}), F_{2}(\mathbf{x}, \mathbf{u})\right] \\
& \text { Subject to : } \begin{cases}g(\mathbf{x}, \mathbf{u})=0 & j=1, \ldots, M \\
h(\mathbf{x}, \mathbf{u}) \leq 0 & k=1, \ldots, K\end{cases}
\end{aligned}
$$

where $F$ is known as the objective vector, $F_{1}$ and $F_{2}$ are the bi-objective functions to optimize, $\mathbf{x}$ is the vector of dependent variables, and $\mathbf{u}$ is the vector of control variables. 
In all optimization problems, several cases in terms of the using of DG are considered namely:

(1) Base case

(2) Case-1: DG only

The objective functions considered in this paper are presented in detail as given below.

a. Maximize the System Loadability within Security Margin

Maximise $F_{2}(\mathbf{x}, \mathbf{u})=\left\{\lambda_{1}\right\}$

Subject to $V L=\sum_{i=1}^{N_{L}} O L L_{i}+\sum_{j=1}^{N_{E}} B V V_{j}$

where $V L$ is the thermal and bus violation limit factor, $O L L_{i}$ and $B V V_{j}$ represent the overloaded line factor and branch the bus voltage violation factor respectively and will be expatiated on later; $N_{L}$ and $N_{E}$ are the total numbers of transmission lines and load buses respectively; and $\lambda_{I}$ is a load parameter of the system, which aims to find the maximum amount of power that the network is able to supply within system security margin.

The load parameter $\lambda_{l}$ in (5) is defined as a function of a load factor $\lambda_{f}[10]$ :

$$
\lambda_{1}=\exp \left[\gamma\left|\lambda_{f}-\lambda_{f}^{\max }\right|\right] \quad \lambda_{f} \in\left[1, \lambda_{f}^{\max }\right]
$$

where $\gamma$ is the coefficient to adjust the slope of the function, and $\lambda_{f}^{\max }$ is the maximal limit of $\lambda_{f}$. The load factor $\lambda_{f}$ reflects the variation of power demands $P_{D i}$ and $Q_{D i}$, which are defined as:

$$
\begin{aligned}
& P_{D i}\left(\lambda_{f}\right)=\lambda_{f} P_{D i} \\
& Q_{D i}\left(\lambda_{f}\right)=\lambda_{f} Q_{D i}
\end{aligned}
$$
load case.

where $i=1, \ldots, N_{D}$ and $N_{D}$ is the total number of power demand buses. $\lambda_{f}=1$ indicates the base

The index of system security state contains two parts. The first part, $O L L_{i}$, relates to the branch loading and penalizes overloads in the lines. The value of $O L L_{i}$ equals to 1 if the $j^{\text {th }}$ branch loading is less than its rating. $O L L_{i}$ increases logarithmly (actual logarithm) with the overload and it can be calculated from:

$$
O L L_{i}= \begin{cases}1 ; & \text { if } P_{i j} \leq P_{i j}^{\max }, \\ \left.\exp \left(\Gamma_{O L L} \mid 1-\frac{P_{i j}}{P_{i j}^{\max }}\right)\right) ; & \text { if } P_{i j} \geq P_{i j}^{\max },\end{cases}
$$

where $P_{i j}$ and $P_{i j}^{\max }$ are the real power flow between buses $i$ and $j$ and the thermal limit for the line between buses $i$ and $j$ respectively. $\Gamma_{O L L}$ is the coefficient which is used to adjust the slope of the exponential function.

The second part $B V V_{j}$ in (9) concerns the voltage levels for each bus of the power network. The value of $B V V_{j}$ is defined as:

$$
B V V_{j}= \begin{cases}1 ; & \text { if } 0.9 \leq V_{\mathrm{b}} \leq 1.1 \\ \exp \left(\Gamma_{B V V}\left|1-V_{b}\right|\right) ; & \text { otherwise }\end{cases}
$$

where $B V V_{j}$ is the bus voltage violation factor at bus $j$ and $\Gamma_{B V V}$ represents the coefficient used to adjust the slope of the exponential function in the above equation. The equation indicates that appropriate voltage magnitudes are close to 1 p.u. Similar to $O L L_{i}$, The value of $B V V_{j}$ equals to 1 if the voltage level falls between the voltage minimal and maximal limits. Outside the range, $B V V_{j}$ increases exponentially with the voltage deviation.

b. Minimization of Real Power Loss of the Transmission Lines

This objective is to minimize the real power loss $\left(\mathrm{P}_{\text {loss }}\right)$ in the transmission lines which can be expressed as [11]:

Optimal Integration of the Renewable Energy to the Grid by Considering Small Signal ... (I Made Wartana) 
Minimize $P_{\text {los }}$

$$
F_{2}(\mathbf{x}, \mathbf{u})=P_{\text {loss }}=\sum_{i=1}^{N} \sum_{j=1}^{N}\left[\alpha_{i j}\left(P_{i} P_{j}+Q_{i} Q_{j}\right)+\beta_{i j}\left(Q_{i} P_{j}+P_{i} Q_{j}\right)\right]
$$

Where

$\alpha_{i j}=\frac{r_{i j}}{V_{i} V_{j}} \cos \left(\delta_{i}-\delta_{j}\right) \quad ; \quad \beta_{i j}==\frac{r_{i j}}{V_{i} V_{j}} \sin \left(\delta_{i}-\delta_{j}\right)$

$V_{i} \angle \delta_{i} \quad$ complex voltage at the bus $i^{\text {th }}$;

$r_{i j}+j x_{i j}=Z_{i j} \quad i j^{\text {th }}$ element of [Zbus] impedance matrix;

$P_{i}$ and $P_{j} \quad$ active power injections at the $i^{\text {th }}$ and $j^{\text {th }}$ buses, respectively;

$Q_{i}$ and $Q_{j} \quad$ reactive power injections at the $i^{\text {th }}$ and $j^{\text {th }}$ buses, respectively;

$\mathrm{N}$ number of buses.

c. Dependent Variables and Control Variables

In the two objective functions, $\mathbf{x}$ is the vector of dependent variables such as slack bus power $P_{G I}$, load bus voltage $V_{m+1 \ldots \ldots .} V_{N b}$, generator reactive power outputs $Q_{G}$, and apparent power flow $S_{k} ; \mathbf{x}$ can be expressed as:

$$
\mathbf{x}^{T}=\left[P_{G 1}, V_{m+1} \ldots V_{N_{b}}, Q_{G_{1}} \ldots Q_{G_{N G}}, S_{1} \ldots S_{N_{L}}\right]
$$

Furthermore, $\mathbf{u}$ is a set of control variables such as generator real power outputs $P_{G}$ except at the slack bus $P_{G 1}$, generator voltages $V_{G}$, the locations of the DG, $L$, and their setting parameters. $\mathbf{u}$ can be expressed as:

$$
\mathbf{u}^{T}=\left[P_{G_{2}} \ldots P_{G_{m}}, P_{D G}, V_{G_{2}} \ldots V_{G_{m}}, L_{1} \ldots L_{N}, \lambda_{f}\right]
$$

where, $N$ is the number of DG to be optimally located. The equality and inequality constraints of the Newton Rhapson power flow problem incorporating DG devices are given bellow.

d. Equality Constraints

These constraints represent the typical load flow equations as follows:

$$
\begin{aligned}
& P_{G_{i}}=P_{D_{i}}+V_{i} \sum_{i=1}^{N_{i}} V_{j}\left(G_{i j} \cos \theta_{i j}-B_{i j} \sin \theta_{i j}\right), \\
& i=1, \ldots N_{0} \\
& Q_{G_{i}}=Q_{D_{i}}+V_{i} \sum_{i=1}^{N_{i}} V_{j}\left(G_{i j} \sin \theta_{i j}-B_{i j} \cos \theta_{i j}\right), \\
& i=1, \ldots N_{P Q}
\end{aligned}
$$

Where $N_{i}$ is the number of buses adjacent to bus $i$ including bus $i, N_{\mathrm{PQ}}$ and $N_{0}$ are the number of $P Q$ buses and total buses excluding slack bus, respectively.

e. Inequality Constraints

The inequality constraints $h(\mathbf{x}, \mathbf{u})$ are the limits of control variables and state variables. Generator active power $P_{G}$, reactive power $Q_{G}$ and voltage $V_{G}$ are restricted by their limits as follows:

$$
\begin{array}{ll}
P_{G_{i}}^{\min } \leq P_{G_{i}} \leq P_{G_{i}}^{\max } & i=1, \ldots \ldots ., N_{G} \\
Q_{G_{i}}^{\min } \leq Q_{G_{i}} \leq Q_{G_{i}}^{\max } & i=1, \ldots \ldots ., N_{G} \\
V_{G_{i}}^{\min } \leq V_{G_{i}} \leq V_{G_{i}}^{\max } & i=1, \ldots \ldots \ldots, N_{G}
\end{array}
$$

The setting parameters of SVC restricted by their limits as follows: 


$$
Q_{P_{i}}^{\min } \leq Q_{P_{i}} \leq Q_{P_{i}}^{\max } \quad i=1, \ldots \ldots, N_{2}
$$

The constraints of load voltages at load buses $V_{L}$ and transmission loading $P_{L}$ are represented as:

$$
\begin{array}{ll}
V_{L_{i}}^{\min } \leq V_{L_{i}} \leq V_{L_{i}}^{\max } & i=1, \ldots \ldots, N_{L} \\
\left|P_{L_{i}}\right| \leq P_{L_{i}}^{\max } & i=1, \ldots \ldots \ldots, N_{E}
\end{array}
$$

The load factor $\lambda_{f}$ is constrained by its limits as:

$$
1 \leq \lambda_{f} \leq \lambda_{f}^{\max }
$$

f. Small Signal Stability Condition

The system used for the small signal stability analysis is a differential algebraic equation (DAE) set [9], in the form:

$$
\begin{aligned}
& \dot{x}=f(\mathbf{x}, \mathbf{y}) \\
& 0=g(\mathbf{x}, \mathbf{y})
\end{aligned}
$$

where, $\mathbf{x}$ is the vector of the state variables and $\mathbf{y}$ the vector of the algebraic variables, which are only voltages amplitudes $\mathrm{V}$ and phases $\theta$. The system state matrix $\mathrm{A}_{\mathrm{s}}$ is thus computed by manipulating the complete Jacobian matrix $A_{c}$, which is defined by the linearization of the DAE system equations (20) as follow [12]:

$$
\left[\begin{array}{l}
\Delta \dot{\mathbf{x}} \\
0
\end{array}\right]=\left[\begin{array}{cc}
\nabla_{x} f & \nabla_{y} f \\
\nabla_{x} g & \nabla_{y} g
\end{array}\right]\left[\begin{array}{l}
\Delta \mathbf{x} \\
\Delta \mathbf{y}
\end{array}\right]=\left[\begin{array}{cc}
F_{x} & F_{y} \\
G_{x} & G_{y}
\end{array}\right]\left[\begin{array}{l}
\Delta \mathbf{x} \\
\Delta \mathbf{y}
\end{array}\right]=\left[A_{c}\right]\left[\begin{array}{l}
\Delta \mathbf{x} \\
\Delta \mathbf{y}
\end{array}\right]
$$

The state matrix $A_{s}$ is simply obtained by eliminating the algebraic variables as follow:

$$
A_{s}=F_{x}-F_{y} G_{y}^{-1} G_{x}
$$

where, $F_{x}, F_{y}, G_{x}, G_{y}$ are Jacobian Matrices as given in (21).

If the complex eigenvalues of the linearized system have negative real parts, then the power system would be able to withstand small disturbances and is thus, considered stable in the small-signal sense. The eigenvalue stability analysis is incorporated in the constraint by the equation (22) in PSAT:

$$
E_{i}\left(F_{x}, F_{y}, G_{y}, G_{x}\right)=0
$$

The eigenvalue based stability assures grid stability under various levels of system loadability.

\subsection{NSGA-II Optimization Principle}

The capabilities of multi-objective genetic algorithms (MOGAs) to explore and discover Pareto optimal fronts on multi-objective optimization problems have been well recognized. It has been shown that MOGAs outperform traditional deterministic methods to this type of problem due to their capacity to explore and combine various solutions to find the Pareto front in a single run. This paper has been implemented a multi-objective optimization technique called the Non-Dominated Sorting Genetic Algorithm II (NSGA-II), which is described in detail by Deb et al. [13].

Once the Pareto optimal set is obtained, it is practical to choose one solution from all solutions that satisfy different goals to some extends. Due to the imprecise nature of the decision maker's (DM) judgment, it is natural to assume that DM may have fuzzy or imprecise nature goals of each objective function [14] Hence, the membership functions are introduced to represents the goals of each objective function; each membership function is defined by the experiences and intuitive knowledge of the decision maker. In this study, a simple linear membership function was considered for each of the objective functions. The membership function is defined as follows [14]: 


$$
\mu_{i}= \begin{cases}1 & 1 \\ \frac{F_{i}^{\max }-F_{i}}{F_{i}^{\max }-F_{i}^{\min }} & F_{i}^{\min }<F_{i}<F_{i}^{\max } \\ 0 & 0\end{cases}
$$

Where $F_{i}^{\text {min }}$ and $F_{i}^{\text {max }}$ are the minimum and the maximum value of the $i^{\text {th }}$ objective function among all non-dominated solutions, respectively. The membership function $\mu$ is varied between 0 and 1 , where $\mu=$ 0 indicates the incompatibility of the solution with the set, while $\mu=1$ means full compatibility. For each non-dominated solution $\mathrm{k}$, the normalized membership function $\mu^{k}$ is calculated as:

$$
\mu^{k}=\frac{\sum_{i=1}^{N_{o b j}} \mu_{i}^{k}}{\sum_{k=1}^{M} \sum_{i=1}^{N_{o b j}} \mu_{i}^{k}}
$$

where, $M$ is the number of non-dominated solutions and $N_{o b j}$ is the number of objective functions. The function $k$ can be considered as a membership function of non-dominated solutions in a fuzzy set, where the solution having the maximum membership in the fuzzy set is considered as the best compromise solution.

\section{RESULTS AND DISCUSSION}

The NSGA II algorithm is carried out in the modified IEEE 14-bus test system [9],[15]. The type of DG incorporated in this simulation is Variable Speed Wind Turbine with DFIG which injects both active and reactive power. The loads are typically represented as constant PQ loads with constant power factor, and increased according to (7) and (8). The DG should be formed at low voltage side, consisting of buses. The number of DG is specified by user, here as equal one and only DG type 3 considered. The parameters of NSGA-II for all optimization cases are summarized in Table 1.

Table 1. NSGA Parameters

\begin{tabular}{cccccc}
\hline Population & Generations & Pool size & Tour Size & $\eta_{\mathrm{c}}$ & $\eta_{\mathrm{m}}$ \\
\hline 100 & 100 & 25 & 2 & 20 & 20 \\
\hline
\end{tabular}

From above condition with population size of 100 and after 100 iterations, 100 dominated solutions are found by the proposed algorithm.

- IEEE 14-bus system

This test system [9],[15] consists of two generators, located at buses-1 and 2; three synchronous compensators are used only for reactive power support at buses-3, 6, and 8 .

The best locations, settings, maximum $S L$ (MSL) and minimum $P_{\text {loss }}$ have been obtained using the NSGA-II technique for each case as given in Tables 2 and 3. The Pareto fronts for the best compromise solutions of all cases for the bi-objective optimizations are also presented in Figs. 1 and 2, respectively.

- Basecase: without DG

\begin{tabular}{|c|c|c|c|c|c|}
\hline & \multirow{2}{*}{$\begin{array}{c}\text { Location } \\
\text { (bus) }\end{array}$} & \multicolumn{2}{|c|}{ Sizing } & \multirow{2}{*}{$\begin{array}{c}\mathrm{SL}\left(\mathrm{F}_{1}\right) \\
(\%)\end{array}$} & \multirow{2}{*}{$\begin{array}{c}\mathrm{P}_{\text {loss }}\left(\mathrm{F}_{2}\right) \\
(\%)\end{array}$} \\
\hline & & MW & MVAR & & \\
\hline Best SL & 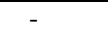 & - & - & 149.59 & 9.274 \\
\hline Best $\mathrm{Pl}_{\text {oss }}$ & - & - & - & 111.51 & 5.627 \\
\hline Best CS & - & - & - & 114.40 & 5.751 \\
\hline
\end{tabular}

Table 2. Load Flow Result of Basecase for Bi-Objective Optimization of IEEE 14-Bus System 


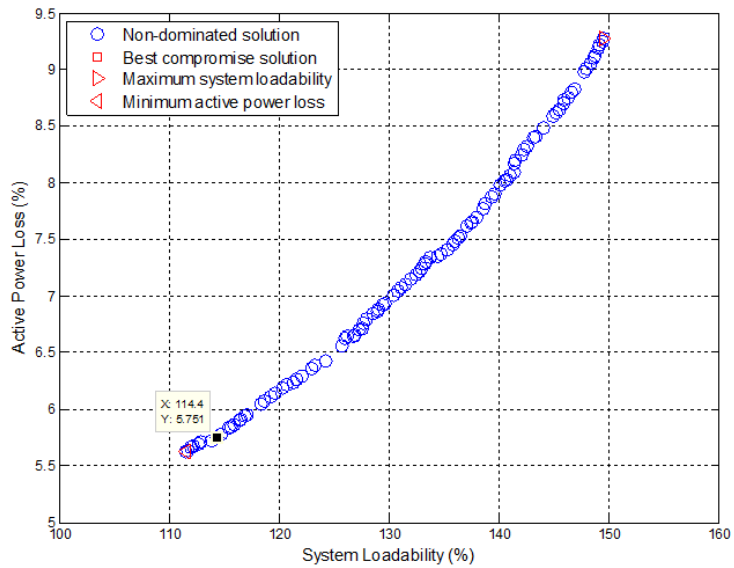

Figure 1. Pareto front of base case for bi-objective optimization of IEEE 14-bus system

\section{- Case-1: With DG}

Figure 2 shows the Pareto front of the optimization problem Case-1, in the objective space: maximizing system loadability (SL) and minimizing real system power loss $\left(\mathrm{P}_{\text {loss }}\right)$. This set of solutions on the non-dominated frontier is used by the decision maker as the input to select a final compromise solution by using the normalized membership function in (25). The obtained results presented in Table 3 indicate that the best compromise solution of configuration plan of DG placement within the system by considering small signal stability constraint is found at bus 5 with size of $49.91 \mathrm{MW}$ and -11.56 MVAR. Moreover, the installation of the DG at bus 8 provides the best SL of $186.1 \%$ as well but with the $\mathrm{P}_{\text {loss }}$ of 0.4885 p.u. (13.87 $\%$ ) which is the highest ones in this case. In addition, this SL is quite large compare with the result obtained in base case as given in Table 2. In the same table, it can be observed that the best $\mathrm{P}_{\text {loss }}$ in this case has been obtained of $8.068 \%$ by installing the DG at bus 14 but it can increase the SL only $129.113 \%$. This SL is the lowest SL the case-1.

The eigenvalue, which represented the stability of system in term of small signal stability at the best compromise solution is depicted in Fig. 3. It is evident that the installation of Wind Turbine assures grid stability with all the eigenvalues in the left hand side of the S-plane during the best compromise solution. Furthermore, the graph does not include the far end stable eigenvalues (real eigenvalue less than -3 ) in the chart.

Table 3. Optimal Placement of DG for bi-objective optimization of IEEE 14-bus System

\begin{tabular}{|c|c|c|c|c|c|}
\hline & \multirow{2}{*}{$\begin{array}{c}\text { Location } \\
\text { (bus) }\end{array}$} & \multicolumn{2}{|c|}{ Sizing } & \multirow{2}{*}{$\begin{array}{c}S L\left(F_{l}\right) \\
(\%)\end{array}$} & \multirow{2}{*}{$\begin{array}{c}P_{\text {loss }}\left(F_{2}\right) \\
(\%)\end{array}$} \\
\hline & & $M W$ & $M V A R$ & & \\
\hline Best $S L$ & 8 & 87.52 & -3.24 & 186.1 & 13.87 \\
\hline Best $P l_{o s s}$ & 14 & 47.47 & -17.34 & 129.113 & 8.068 \\
\hline Best CS & 5 & 49.91 & -11.46 & 134.1 & 8.184 \\
\hline
\end{tabular}

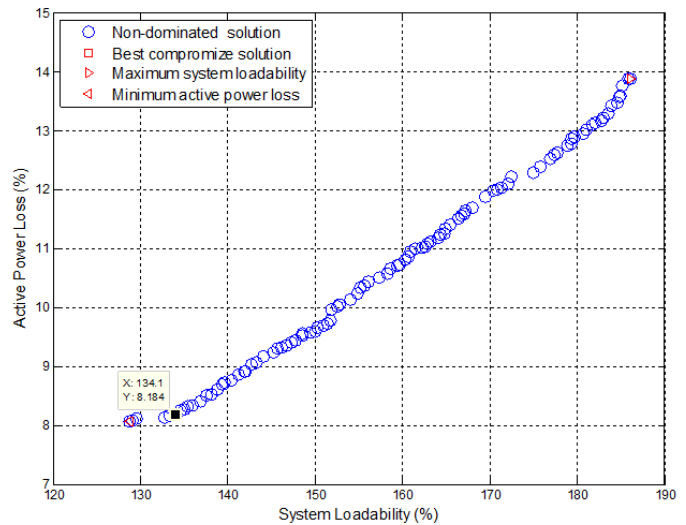

Figure 2. Pareto front of optimal location and size for bi-objective optimization of IEEE 14-bus system 


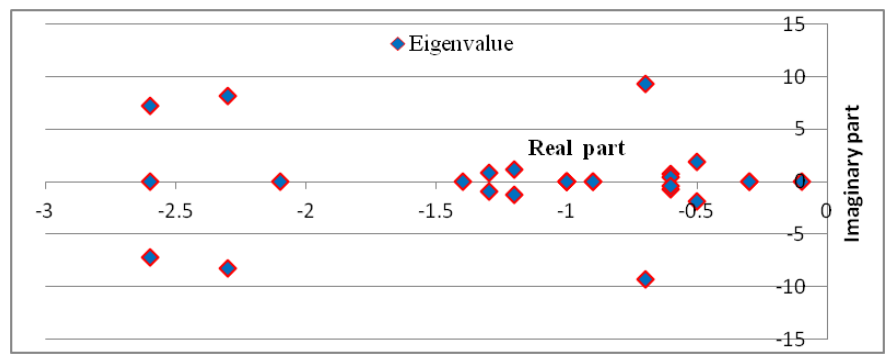

Figure 3. Eigenvalue of optimal location and size for bi-objective optimization of IEEE 14-bus system

The results obtained by applying the NSG-II technique for IEEE 14-bus system is compared with the results reported in [4], [5] and [7] as shown in Table 4. From this table, it can be seen that installing a DG at the suitable location in the system, the maximum SL (MSL), size of DG, and $P_{\text {loss }}$ obtained by proposed method is $134.1 \%, 47.47 \mathrm{MW}$ and $26.9 \mathrm{MW}$, respectively.

When compare with [5], the proposed results need more 31.47 MW size of DG to increase system loadability of $34.1 \%$ but $P_{\text {loss }}$ is higher $15.2 \mathrm{MW}$ than the result in [5]. With the location at bus 14, the result obtained in [7] requires less 7.47 MW size of DG compare with the proposed method to find $P_{\text {loss }}$ of 28.83 MW. The $P_{\text {loss }}$ is higher $7.17 \%$ compared with the proposed method. Whereas the result reported in [4], it needed $8 \mathrm{DG}$ with the total size of $217.91 \mathrm{MW}$ to find the $P_{\text {loss }}$ of $4.84 \mathrm{MW}$ without increasing the system loadability. These size are increasing quite large $(359 \%)$ with $P_{\text {loss }}$ decreased only 22.06 MW compared with the obtained result in this work. Moreover, maximization of system loadability by considering all stability constraints of the standard IEEE 14-bus test system are not incorporated in [4], [5] and [7]. Therefore, the suggested approach in this paper has been found as more suitable and practical compared with reported literature for similar work.

Table 4. Optimal location, Max SL. Size, $P_{\text {loss }}$ and Maximum Number of DG (N) in IEEE 14-bus System

\begin{tabular}{ccccccc}
\hline $\begin{array}{c}\text { Obtained } \\
\text { Results of } \\
\text { DG placement }\end{array}$ & $\mathrm{N}$ & $\begin{array}{c}\text { MSL } \\
(\%)\end{array}$ & $\begin{array}{c}\text { Total Size } \\
(\mathrm{MW})\end{array}$ & $\begin{array}{c}\mathrm{P}_{\text {loss }} \\
(\mathrm{MW})\end{array}$ & $\begin{array}{c}\text { Optimal Locations } \\
\text { (bus) }\end{array}$ & $\begin{array}{c}\text { Considered } \\
\text { stability } \\
\text { (section 2.2. f) }\end{array}$ \\
\hline Proposed method & 1 & 134.1 & 47.47 & 26.9 & 5 & Yes \\
{$[4]$} & 8 & 100 & 217.91 & 4.84 & $3,7,9,10,11,12,13$, and 14 \\
[5] & 1 & 100 & 16 & 11.70 & 8 & No \\
{$[7]$} & 2 & 100 & 40 & 28.83 & 14 & No
\end{tabular}

\section{CONCLUSION}

A Novel approach based on NSGA-II has been presented in this work and applied to optimal location, setting and sizing of one type renewable energy represented by a DG in power network by considering not only security system but also small signal stability constraint. The problem is formulated as a real mixed continuous integer bi-objective optimization problem, where two conflicting objective problems have been simultaneously considered viz.: maximizing system loadability (SL) and minimizing real power losses $\left(\mathrm{P}_{\text {loss }}\right)$. In each case, the optimal location, setting and sizing of the DG are performed for several uses of the devices by considering security and small signal stability constraints. Moreover, a fuzzy based mechanism is employed to extract the best compromise solution from the Pareto front. The results show that NSGA-II provides well distributed non dominated solutions and well exploration of the research space.

\section{ACKNOWLEDGEMENTS}

The authors express their gratitude to the Ministry of Research, Technology and Higher Education of the Republic of Indonesia and National Institute of Technology (ITN) Malang for providing grant to conduct this research.

\section{REFERENCES}

[1] M. N. Lakhoua, N. Walid, and C. Atef, "System Analysis of a Hybrid Renewable Energy System," International Journal of Electrical and Computer Engineering, vol. 4, p. 343, 2014. 
[2] H. E. A. Talaat and E. Al-Ammar, "Optimal allocation and sizing of Distributed Generation in distribution networks using Genetic Algorithms," in Electrical Power Quality and Utilisation (EPQU), 2011 11th International Conference on, 2011, pp. 1-6.

[3] H. Shahinzadeh, G. B. Gharehpetian, S. H. Fathi, and S. M. Nasr-Azadani, "Optimal Planning of an Off-grid Electricity Generation with Renewable Energy Resources using the HOMER Software," International Journal of Power Electronics and Drive Systems, vol. 6, p. 137, 2015.

[4] N. K. Aryani, M. Abdillah, I. M. Y. Negara, and A. Soeprijanto, "Optimal placement and sizing of Distributed Generation using Quantum Genetic Algorithm for reducing losses and improving voltage profile," in TENCON 2011 - 2011 IEEE Region 10 Conference, 2011, pp. 108-112.

[5] S. Ghosh, S. P. Ghoshal, and S. Ghosh, "Optimal sizing and placement of distributed generation in a network system," International Journal of Electrical Power \&amp; Energy Systems, vol. 32, pp. 849-856, 2010.

[6] A. Uniyal and A. Kumar, "Comparison of optimal DG placement using CSA, GSA, PSO and GA for minimum real power loss in radial distribution system," in 2016 IEEE 6th International Conference on Power Systems (ICPS), 2016, pp. 1-6.

[7] C. Xi and G. Wenzhong, "Effects of Distributed Generation on power loss, loadability and stability," in Southeastcon, 2008. IEEE, 2008, pp. 468-473.

[8] H. Jadhav and R. Roy, "A comprehensive review on the grid integration of doubly fed induction generator," International Journal of Electrical Power \& Energy Systems, vol. 49, pp. 8-18, 2013.

[9] F. Milano, "An Open Source Power System Analysis Toolbox," Power Systems, IEEE Transactions on, vol. 20, pp. 1199-1206, 2005.

[10] Z. Lu and M. S. Li, Jiang, L., Wu, Q. H., "Optimal allocation of FACTS devices with multiple objectives achieved by bacterial swarming algorithm," in Power and Energy Society General Meeting - Conversion and Delivery of Electrical Energy in the 21 st Century, 2008 IEEE, 2008, pp. 1-7.

[11] OJ Petinrin, M Shaaban, "Overcoming Challenges of Renewable Energy on Future Smart Grid," TELKOMNIKA Telecommunication, Computing, Electronics and Control, vol. 10, no. 2, pp. 229-234, 2012.

[12] S. Lamichhane and N. Mithulananthan, "Possible impact of large scale wind energy integration on small signal stability," in 2015 IEEE PES Asia-Pacific Power and Energy Engineering Conference (APPEEC), 2015, pp. 1-5.

[13] K. Deb, A. Pratap, S. Agarwal, and T. Meyarivan, "A fast and elitist multiobjective genetic algorithm: NSGA-II," Evolutionary Computation, IEEE Transactions on, vol. 6, pp. 182-197, 2002.

[14] R. Benabid and M. Boudour, Abido, M. A., "Optimal location and setting of SVC and TCSC devices using nondominated sorting particle swarm optimization," Electric Power Systems Research, vol. 79, pp. 1668-1677, 2009.

[15] R. D. Zimmerman, S. n. Murillo, C. E., and R. J. Thomas, "MATPOWER: Steady-State Operations, Planning, and Analysis Tools for Power Systems Research and Education," IEEE Transactions on Evolutionary Computation, vol. 26, pp. 12-19, 2011.

\section{BIOGRAPHIES OF AUTHORS}
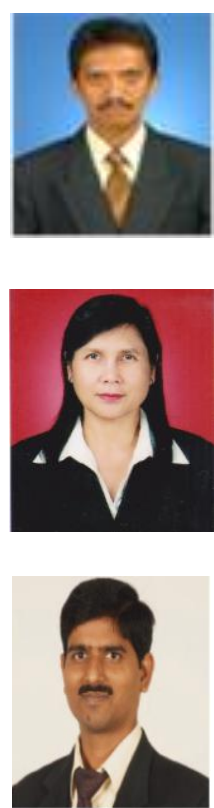

I Made Wartana received his B.Eng. and M.Eng. from Electrical Engineering Department, National Institute of Technology (ITN) Malang, East of Java, Indonesia and Bandung Institute of Technology (ITB), West of Java, Indonesia in 1986 and 1994, respectively. He has been a lecturer at ITN Malang, Indonesia since 1992. He also received his Dr. Eng. from the Asian Institute of Technology (AIT), Thailand in 2087. His research interests are in application of FACTS controllers in power grid, AI application to power system, and in grid integration of renewable.

Ni Putu Agustini received her B.Eng. and M.Eng. from Electrical Engineering Department, National Institute of Technology (ITN) Malang, East of Java, Indonesia and Brawijaya University (UB), East of Java, Indonesia in 1986 and 2008, respectively. She has been a lecturer at ITN Malang, Indonesia since 2004. Her research interests are in integration of renewable energy in power grid, and reliability application to power system.

Dr. Jai Govind Singh (M'10) received his M. Tech. and Ph. D. degrees, all in Electrical Engineering, from IIT Rookree and IIT Kanpur, respectively. He is currently Associate Professor in the energy field of study, Asian Institute of Technology, Thailand. His research interests include power system operation and control, FACTS, power sector deregulation and power system planning, integration of renewable energy resources into power system and power distribution system. 